\title{
Demonstration of Distributed TDMA MAC Protocol Implementation with OLSR on Linux Enriched WARP
}

\author{
Hannu Tuomivaara \\ Centre for Wireless \\ Communications, \\ University of Oulu \\ Erkki Koiso-Kanttila Street 3 \\ 90570 Oulu, Finland \\ hannu.tuomivaara \\ @ee.oulu.fi
}

\author{
Matti Raustia \\ Centre for Wireless \\ Communications, \\ University of Oulu \\ Erkki Koiso-Kanttila Street 3 \\ 90570 Oulu, Finland \\ matti.raustia@ee.oulu.fi
}

\author{
Markku Jokinen \\ Centre for Wireless \\ Communications, \\ University of Oulu \\ Erkki Koiso-Kanttila Street 3 \\ 90570 Oulu, Finland \\ markku.jokinen@ee.oulu.fi
}

\begin{abstract}
In this demo we present software extension for the Wireless open Access Research Platform (WARP), named as Linux Enriched WARP (LE-WARP). The objective of the LEWARP is twofold. First, to make the WARP more independent from external systems, e.g., need for external PC to be part of the WARP's network layer. This is achieved by introducing Linux OS to the platform. The second objective is to utilize a secondary network for coordinated statistics gathering from the WARPs. To demonstrate these features of LE-WARP, a prototype implementation consisting of distributed Time Division Multiple Access (TDMA) based MAC protocol with distributed time synchronization algorithm and the Optimized Link State Routing (OLSR) protocol for ad hoc networks is presented. The demonstration highlights the LE-WARP's ability to monitor the multihop test network consisting four nodes while utilizing the presented prototype implementation.
\end{abstract}

\section{Categories and Subject Descriptors}

B.4.1 [Data Communications Devices]: Transmitters**; Receivers (e.g., voice, data, image); C.2.3 [Network Operations]: Network monitoring

\section{General Terms}

Experimentation

\section{Keywords}

Linux, OLSR, TDMA, WARP, ad hoc, synchronization

\section{INTRODUCTION}

The ongoing trend in research community is to give more emphasis on prototyping of new algorithms and protocols. Usually this is realized by implementing new algorithms and protocols to research platforms, like the WARP [3]. The WARP is built from ground up to allow clean-slate prototyping of PHY and MAC protocols designed for wireless networks. As the prototyping phase advance from link to link tests to network scale tests, the requirements for the

Copyright is held by the author/owner(s).

WiNTECH'09, September 21, 2009, Beijing, China.

ACM 978-1-60558-740-0/09/09. platform increases, e.g., need for dynamic routing becomes inevitable. Thus, without routing protocol it is difficult to test a new MAC protocol in a dynamic test network. While implementing distributed TDMA based MAC protocol on the WARP we felt the need to have fully functional network layer on WARP. As we had to be able to utilize dynamic routing when testing our implementation in ad hoc scenarios. In addition, method for coordinated statistics gathering from the platforms was required. As the validation and debugging of a prototyped MAC protocol on a network scale was discovered to be impractical without any coordination or centralized statistics gathering entity.

In the following we present the LE-WARP software enchantment to fulfill above mentioned requirements. As an example of prototype implementation a distributed TDMA based MAC protocol is presented with the OLSR routing protocol and lastly the demo is presented that shows the prototype implementation functioning on top of the LE-WARP.

\section{LINUX ENRICHED WARP}

The LE-WARP software extension is a combination of novel dual core system design and a software framework for enabling an independent secondary network for monitoring and statistics gathering of the prototyped network.

In system design perspective, as two PowerPC cores already existed on the FPGA fabric of the WARP. We decided to utilize a novel dual core design, architecture is shown in Fig. 1. In this dual core design the PHY controlling and MAC layer processing is done by PowerPC core 0 . The design follows the OFDM reference design [1] in some extent, e.g, it utilizes the same physical layer and the software framework as the OFDM reference design. Therefore, the same design tools can be used for implementing the PHY/MAC layer protocols. The higher OSI-layer processing is carried out in PowerPC core 1 by the Linux operating system (OS). The Linux OS is based on kernel version 2.6.28 and it was further customized for the WARP. Additional kernel drivers were designed to enable networking capabilities of the WARP and the inter-communication between the PowerPC cores. The inter-communication between the two cores is carried out via buffering scheme where data, control, and statistical information flows are separated, thus making the design robust against failures. This inter-communication enables monitoring of the PHY and MAC layer implementations from the Linux OS, via statistics logging unit that 


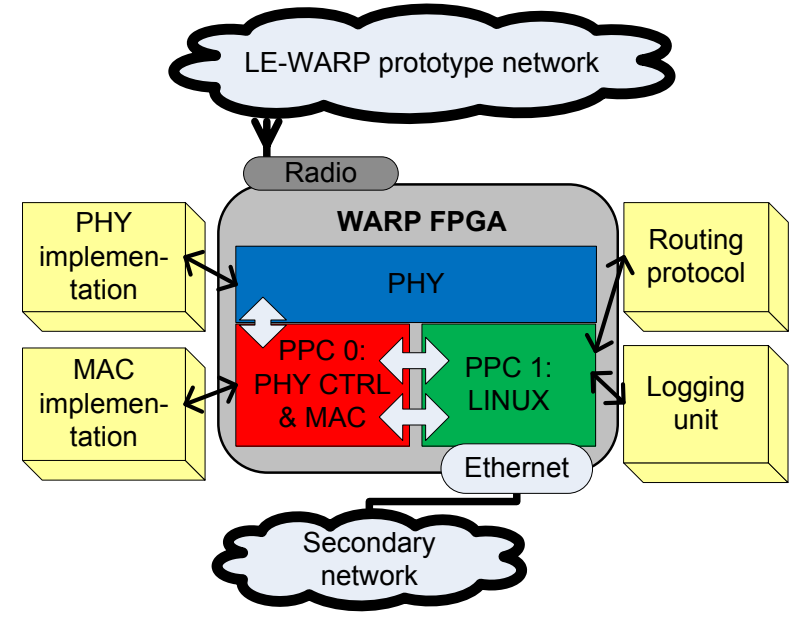

Figure 1: The LE-WARP architecture.

writes statistics gather from $\mathrm{PHY} / \mathrm{MAC}$ side to a file in Linux. The Linux OS also brings many interesting opensource applications available for the LE-WARP.

The LE-WARP introduces two main features to extend the existing WARP. First, the Linux OS adds fully functional network layer with IPv4 stack and application layer to it. This feature makes WARP become more like selfsustained system with configurable in PHY, MAC and network layers. Second, the LE-WARP enables a framework for statistics gathering from nodes on the prototype network to a remote host via secondary network. The remote host performs as a coordinator for statistics gathering from the LE-WARP nodes as well as a statistics data gathering storage for the whole network. The secondary network does not utilize the WARP's radio interface only the platform's Ethernet connection, hence, it does not interfere with the prototyping of PHY or MAC protocols.

\section{PROTOTYPE IMPLEMENTATION}

To demonstrate the LE-WARP's capabilities with already existing OFDM physical layer [1] a distributed TDMA based MAC protocol [5] and a distributed time synchronization algorithm [6] with the OLSR routing protocol [2] was implemented on top of the LE-WARP. The distributed TDMA based MAC protocol has fairly simple design to it; first, joining node acquires local neighborhood information from surrounding nodes. The local neighborhood information consists of neighborhood connectivety information and slot assignments from two-hops vicinity from particular node's point of view. After gathering sufficient local information particular node may allocate a free time slot and may start data transmission with the nodes on the network.

Time synchronization is one of the major challenges in distributed TDMA based MAC protocol designed for ad hoc network since the access to the medium is divided in time among the nodes the network. Hence, a distributed synchronization algorithm is included to be a part of the distributed TDMA based MAC protocol design.

The routing algorithm employed in this implementation is OLSR, the implementation of it is known as OLSR daemon (OLSRd) [4]. The OLSRd is highly portable implementation that allows routing for any network equipment with functional IP stack.
The protoype implementation is divided into building blocks. They are time synchronization unit, local neighborhood information unit, transmission scheduling unit. Ready available OLSRd routing protocol was embedded as it is into Linux OS' network layer. All these building blocks are designed to emit statistical information that is saved locally to a log file by the LE-WARP node. Then this information is gathered for further processing by remote host via independent secondary network that connects remote host and the LE-WARP nodes together.

\section{DEMONSTRATIONS}

We demonstrate the LE-WARP's two main features with prototype implementation. The demo is divided to two separate sections. The first section of the demo shows how network consisting of four LE-WARP embedded platforms with prototype implementation form a functional multihop network. While statistics from the MAC and network layer building blocks are gathered via independent secondary network to a remote host, in this case to a laptop. This statistical information from the nodes is displayed on a laptop trough a graphical user interface (GUI) program designed for this purpose.

The second section of the demo emphasize the robustness and adaptivity of the prototype implementation in a varying network topology. The ability of MAC and routing protocols to adapt to link breaks caused by topology changes are shown while the end-user application is running on the laptops connected to two of the LE-WARP enhanced platforms. The end-user application is a video conference executed over multiple hops. At the same time, the statistics from nodes on the current network can be followed from the GUI.

\section{ACKNOWLEDGMENTS}

The authors would like to thank Rice University for the support with the WARP boards.

\section{REFERENCES}

[1] WARP Repository (24.7.2009) url:http://warp.rice.edu/trac.

[2] T. Clausen and P. Jacquet. Optimized Link State Routing Protocol (OLSR). RFC 3626 (Experimental), Oct. 2003.

[3] A. Khattab, J. Camp, C. Hunter, P. Murphy, A. Sabharwal, and E. W. Knightly. WARP: a flexible platform for clean-slate wireless medium access protocol design. SIGMOBILE Mob. Comput. Commun. Rev., 12(1):56-58, 2008.

[4] A. Tonnesen. Impementing and Extending the Optimized Link State Routing Protocol. Master's thesis, Department of Informatics, University of Oslo, 2004.

[5] H. Tuomivaara. Real-Time Implementation of A Distributed TDMA based MAC Protocol. Master's thesis, University of Oulu, CWC, to be published.

[6] T. Vanninen, M. Raustia, H. Saarnisaari, and J. Iinatti. Frequency hopping mobile ad hoc and sensor network synchronization. In Military Communications Conference, 2008. MILCOM 2008. IEEE, pages 1-7, Nov. 2008. 